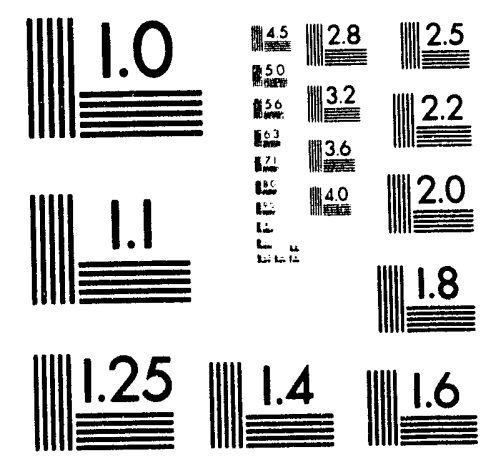



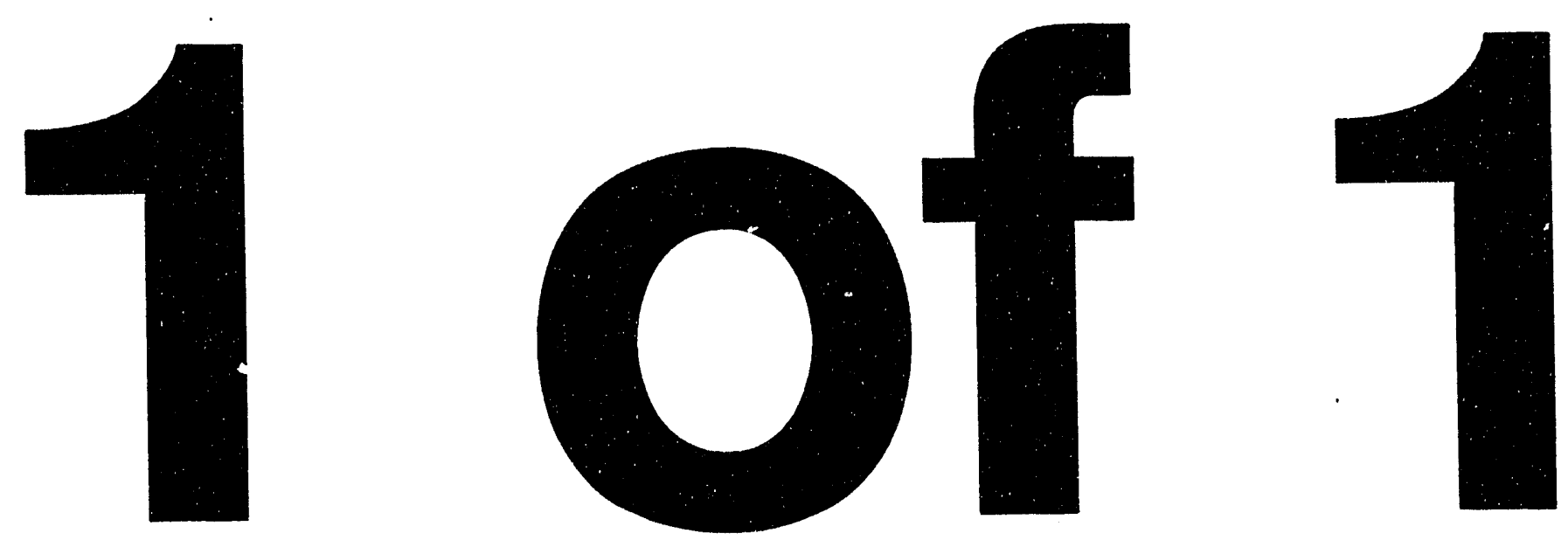


\title{
Object-Oriented DFD Models to Present the Functional and Behavioral Views
}

\author{
Amelia Maxted \\ Advance Information Systems Department \\ Sandia National Laboratories \\ Albuquerque, NM 87185-5800
}

\begin{abstract}
- Abstract
An object-oriented methodology is presented that is based on two sets of Data Flow Diagrams (DFDs): one for the functional view, and one for the behavioral view. The functional view presents the information flow between shared objects. These objects map to the classes identified in the structural view (e.g., Information Model). The behavioral view presents the flow of information between control components and relates these components their state models. Components appearing in multiple views provide a bridge between the views. The top-down hierarchical nature of the DFDs provide a needed overview or road map through the software system.
\end{abstract}




\subsection{Introduction}

This paper presents an object-oriented design methodology that refines today's design methodologies. The methodology is built upon the 3 views of a software system: structural, functional and behavioral as presented by Rumbaugh [1]. Information Models, as presented by Shlaer/Mellor[2], are a standard tool for presenting the structural view. Data Flow Diagram techniques to present the functional and behavioral views are presented herein. These DFDs differ in small but significant ways from those appearing in the literature, Rumbaugh[1] and Ward[3, 4].

The methodology was developed for and has been applied to a satellite ground system. This satellite ground system consists of $100 \mathrm{k}$ lines of application Ada code built upon an in-house repository of 50k lines of Ada code. The satellite ground system was funded by the Department of Defense.

\subsection{Motivation}

The structural view detailing the classes that define a software system lacks an overview. Data Flow Diagrams (DFDs), with their hierarchical nature, present system overviews. However, most DFD techniques of today do not map well to object-oriented systems. A DFD technique that decomposes into shared objects, which are instances of a subset of the classes identified by the structural view is needed. Even the flows should map to the class of data that flows between shared objects. This would result in a set of DFDs that provided an overview of the structural view, identifying the major classes.

Most DFD techniques intertwine two stories, the story of the information flowing between shared object methods and the story told by the control components that move the information between these shared objects. Intertwining the two stories makes it difficult to differentiate the players. A DFD technique that separates these two stories promises a simpler model of the software system. A DFD technique illustrating the information flow between the control components would provide a valuable overview of the behavioral view. 
Introduction

\subsection{Summary}

The DFDs that present the functional view decompose into shared objects. These objects are instances of a subset of the classes identified in the structural view. The DFD flows map to classes, labeled with the type of data that flows between two shared objects. This functional view identifies stand-alone transform software components. This functional view illustrates the interaction between the shared objects and these transforms.

The DFDs that present the behavioral view decompose into the control software components ${ }^{1}$. These control components are responsible for moving data between the shared objects and transforms. This behavioral view illustrates the flow of data between these control components. State models, as presented by Shlaer/Mellor[5], are employed to describe the behavior of the control components as well as the behavior of the shared objects.

The three views are abstractions. Each present overlapping subsets of the software components. The three views together identify all the software components composing a system. The DFDs presented herein provide a hierarchical overview of the software system supplementing the structural view, which lacks an overview. The abstract nature of the views along with the hierarchical nature of the DFDs present the software system in an easy to comprehend manner. The DFDs provide a road map to the software system allowing the customer and developer alike to grasp an overview of the system as well as to focus on a small number of software components.

\subsection{Scope}

The methodology, described in Section 2.0, presents software categories, the dual DFDs and the design document. An example of a sensor monitoring system that employs this methodology is presented in Section 3.0. The methodology is summarized in Section 4.0.

1. Active objects, presented by Shlaer/Mellor[2], do not have separate control components. In such a solution, the objects are responsible for pushing and pulling their own data. 


\subsection{The Methodology}

The methodology presented herein focuses on object-oriented design. Software components are code units that compose a software system. The design results in the identification of most of the software components. Categories of software components are presented in Section 2.1. These categories define the composition of the software component and the role the component plays in the DFDs. The functional and behavioral views and the object and control DFDs that illustrate these views are presented in Section 2.2. The design document that presents the DFDs is described in Section 2.3. An overview of the design methodology is presented in Section 2.4.

\subsection{The Categories}

Seven major categories of software components have been identified.

1. Collections - Group fundamental types defining a domain. Collection exported types are subtypes or derived types of fundamental types (not private types). Few subprograms are exported.

2. Utility Abstract Data Types (ADTs) - Provide sharable tools. Utility ADTs export a type that is not specific to the application domain (e.g, data structures). Subprograms are provided to construct objects of this type and to extract information from objects of this type.

3. Definition Abstract Data Types (ADTs) - Define the application domain data definition ADTs export a private type. Subprograms to construct objects of this type and to extract information from objects of this type are exported.

4. Abstract Data Objects (ADOs) - Maintain a single sharable copy of an object. ADOs define a single instance of a class (ADT). An ADO software component is defined only when the object is to be shared. An object may be shared between control software components or even between invocations of a single control component. ADOs export subprograms that provide synchronized access to the object. Objects stored in disk files and shared memory are easily identified ADOs. 
The Methodology

ADOs do not export the type of the object that they are maintaining. They provide subprograms to add information to and extract information from the state object.

5. Transforms - Transform data from one definition ADT to another.

- Transforms as part of ADTs - Transforms may be incorporated as part of a definition ADT. The construct functions transform imported types to the exported type. The extract functions transform the exported type to imported types.

- Transforms as part of ADOs - Transforms may be included as part of an ADO. Transforms incorporated as part of an ADO are identified when the ADO input is not the same type as the ADO output.

- Transform Software Components - Transforms software components convert between imported types. Transforms and their inverse are packaged together.

6. Actors - Move Abstract Data Type (ADT) data between ADOs and transforms. Actors provide logical functional control. Actors are subprograms. Concurrent mechanisms such as task rendezvous and operating system stream communication are not accessed by the actors. Actor inputs and outputs are the subprogram invocation parameters. By not accessing the concurrent mechanisms directly, the actor remains a logical solution to the control problem and is therefore less subject to change.

7. Threads-of-control - Sequentialize one or more actors. Threads are physical asynchronous mechanisms such as Ada tasks or mainline operating system programs. They employ a physical stream mechanism for input/output, e.g., task rendezvous or Unix pipe. Asynchronous data sources suggest multiple threads. 
The mapping from these categories to the taxonomy presented by Frankel[6] is:

1. Definition Packages - Collections,

2. ADT Packages - Definition ADTs and Utility ADTs,

3. Flow Packages - ADOs that manage stream communication,

4. Algorithmic Packages - Transforms,

5. Entity Packages - ADOs, and

6. Active Task Packages - Threads-of-Control.

A distinction between ADTs has been made herein because definition ADTs have a definite role in the DFDs but utility ADTs do not. Actors, the logical control software components, have been introduced to improve the immutability of the control components. A logical control solution can be designed before the physical constraints are applied to the solution. Employing categories provide additional metrics to evaluate and improve the quality of the software product. Such metrics are a subject of future work. Judicious use of categories result in a more singular solution composed of immutable software components with a higher potential reuse.

\subsection{Functional and Behavioral Views}

Both the functional and behavioral views are illustrated in the cartoon on FIGURE 1. This cartoon illustrates two ADOs: A Shelf of Vases and a Trash Can. Definition ADTs in and out of these ADOs are illustrated and labeled as, Vase and Trash. A transform is introduced to transform data of type Vase to data of type Trash. This transform connects the ADOs. A single flow path flowing from the shelf of vases through the transform to the trash can is illustrated. Two Actors have been identified to move data along the flow path: Smasher and Cleaner. A Thread-of-Control sequentializing the actors is also illustrated. Utility ADTs and collections are not illustrated. The functional view focuses on the 
The Methodology

story told by the ADOs and transforms. The behavioral view focuses on the story told by the actors and threads. Separating the two views result in two significantly simpler views. The two views tell the same story from two very different points of view. Combined with the structural view, these provide a complete description of the software system.

\subsubsection{The Functional View - Object DFDs}

The object DFDs illustrate the functional view. This view is composed of most ADOs. ADOs that are NOT presented include ADOs that are an artifact of the physical solution such as a stream interface manager and ADOs that implement the control solution such as a state model manager. This view illustrates the flow of data between the ADOs. Control components are not included in the object DFDs, therefore control flows are suppressed. The ADOs are represented as bubbles. Definition ADTs that define the data that flow in and out of the ADOs are represented as arrows in and out of the ADO bubble labeled with the definition ADT type. The ADO input and output flows are interconnected to illustrate the flow of data between ADOs. Tranisforms are introduced into the DFDs in order to resolve data discontinuities between ADOs. Stand-alone transforms are represented as boxes. Though boxes can be used to represent transforms that are part of ADTs, the author recommends using an anchor point. For an AD'T construct function, multiple arrows, labeled with their respective ADT types, flow into the point. A single arrow flows out of the point, labeled with the ADT type. For an ADT extract function, a single arrow labeled with the ADT type flows into the point. Multiple arrows, labeled with their respective ADT types, flow out of the point. The object DFD for the cartoon of FIGURE 1, is illustrated in FIGURE 2. Each ADO manages state and therefore should appear only once on the DFD. The transforms and the definition ADTs are not associated with state. The same transform may appear any number of times on the data flow. Any number of flows may be labeled with the same ADT name.

The functionality of the ADOs could be illustrated by decomposing the ADO bubble into a DFD where the ADO state is illustrated as a "file" and the ADO functions (methods) are illustrated as 
"bubbles" accessing the "file". This additional level of decomposition may help the reader in understanding the similarities between the object DFD and more traditional DFD techniques such as those presented in Rumbaugh[1]. The author prefers to decompose the object into a textual and graphical description that includes illustrations of the object methods such as Booch's Module Diagram, Booch[7]. Such a decomposition illustrates the same functionality but highlights the rigid object boundary surrounding the ADO.

\subsubsection{The Behavioral View - Control DFDs}

The control DFDs illustrate the behavioral view. This view identifies the actors and threads and illustrates the flow of data between these control software components. Study of the control mechanisms begins with the object DFDs. Flow paths are identified on these object DFDs. A flow path begins and ends on externals and/or ADOs. Flow paths may incorporate any number of transforms. Flow paths may have any number of starting and ending flows. Actors implement these flow paths. There is at lease one actor for each flow path. The control DFDs for the cartoon of FIGURE 1 is illustrated on

\section{FIGURE 3.}

Actors are logical solutions to the control problem. Initially the control DFDs illustrate only this logical solution. Each actor is represented as a bubble. The actor inputs and outputs are illustrated by flows. The flows are labeled with the definition ADT that defines the type of data flowing in and out of the actors. These flows are interconnected to illustrate the flow of data between actors. Actors also share data via ADOs. ADOs shared between actors are illustrated using the structured analysis file symbol. The flows in and out of the ADOs may be labeled with the definition ADT that defines the type of data flowing through the ADO. The description of the actors describes their state model implementations. Combining the Control DFDs with these state models results in a nested set of state models that describe the software behavior. 


\section{The Methodology}

\subsubsection{The DFD Hierarchy}

Both sets of DFDs decompose from the same context diagram. The bottom level flows are identified before the hierarchy decomposing the system into these flows is introduced. The hierarchy is composed by grouping components (objects and transforms or actors) that operate on the same type of definition ADTs or that work together to get a job done into a composite bubble. The context diagram bubble decomposes into composite bubbles that decompose into other composite bubbles or into the components.

Initially the control DFDs decompose into actors only. When the physical control solution is identified, the control DFDs are modified to decompose into threads. The threads then decompose into the actors they sequentialize.

\subsubsection{Labeling the DFD flows}

The ADT names are not qualified by the functionality applied, in contrast to the convention used in structured analysis (SA). The object oriented DFD literature varies as to how the DFD flows are labeled. Herein the flows are labeled with the definition ADT name only. The extract and construct subprogram names implied by the flow end-points are suppressed. The reasons are:

There is not a one-to-one mapping between flow endpoints and subprograms. Multiple flows may be synchronized into a single subprogram with multiple parameters.

By not qualifying the flow, the number of unique flows is greatly reduced resulting an a smaller data dictionary. The smaller data dictionary is easier to maintain and to use. Admittedly, suppressing the functionality from the DFDs make them more difficult to read. The author suggests appending the flow names with descriptive information within parenthesis, as needed, to replace the important qualifying information. The parenthesis annotations should be included to restore readability to the DFDs. 
The Methodology

\subsection{The Design Document}

A design document presenting both sets of DFDs provides a top-level view of the software. In addition to the DFDs, the design document includes:

1. The Object DFD Specifications - Describing each ADO and stand-alone transform identified in the Object DFDs. This description includes both textual and graphical descriptions of the ADO.

2. The Object DFD Data Dictionary - Each flow identified in the Object DFD is defined.

3. The Control DFD Specifications - Describing each thread and actor identified in the Control DFDs This description includes both textual and graphical descriptions (e.g, state models).

4. The Control DFD Data Dictionary - Each flow and each ADO identified in the Control DFDs is defined ${ }^{1}$.

The DFD hierarchy introduces composite flows. These flows are convenient groupings of definition ADTs that simplify the DFDs. The composite flows do not indicate synchronization of the definition ADTs. The data dictionary includes the decomposition of these composite flows into definition ADTs. The data dictionary description of the definition ADTs should be a one sentence textual descriptions. For the composition of the definition ADTs the reader is referred to the structural view.

An additional level of abstraction is achieved in that components are described in differing levels of details. The description of the ADOs includes the specification but not the implementation of the ADO. The description of a definition ADT is limited to a one line textual description. The detail available in the design document increases from preliminary design through delivery as the knowledge on the components increase, but the design document remains an abstraction. It provides an easy to comprehend road map through the delivered software system.

1. It is reasonable to combine the two data dictionaries into a single data dictionary. 
The Methodology

\subsection{The Method}

A method to generate a top-level abstract view of an object-oriented design has been presented. This method is employed at the start of software desigrs. The steps that a designer employing this method might take to design the software include:

1. Categorize Software Components - Classes are categorized as definition ADTs if they define the application data flowing through the system. Instances of these classes are categorizud as ADOs if they are shared objects.

2. Construct the Object DFDs - The Object DFDs, presenting the functional view are constructed.

3. Construct the Control DFDs - The Control DFDs, presenting the behavioral view are constructed.

4. Map Requirements on to the Software Components - Interface requirements are mapped onto the interface definition ADTs (the ADTs appearing on the context diagram). Functional requirements are mapped to the ADOs and transforms composing the object view. These same requirements are mapped on to the actors. Threads inherit the requirements from the actors that they serialize. The mapped requirements are included in the DFD specifications.

5. Describe Software Components - The description varies depending on the component category. The description of an $\mathrm{ADO}$ identifies requirements mapped to the component, and identifies the functionality of the ADO. The description of an actor or thread identifies requirements mapped to the component, and describes the state model implemented by the actor/thread. The description of a definition ADT is a one line textual description.

6. Complete the Structural View - Information modeling and domain analysis results in the identification of all the software components including utility ADTs and collections. 


\subsection{Sensor Monitoring System Example}

\subsection{Example Requirements}

Consider the simplified example of a software system that receives monitor measurements from a remote sensor. The remote sensor reports via monitors its current environment (e.g. temperature), its configuration and its heaith (reporting any detected anomalies). Requirements are:

- Periodically, a monitor set is issued by the sensor.

A set of range limit values is defined for each monitor. The limit values indicate the boundaries between normal and abnormal value bands for each monitor value.

The operator may individually change the limit values for any monitor.

A new set of monitor values is received from the sensor every 30 seconds.

○

When a monitor set is received the monitors are compared against the limit values. A change in band report is issued to the operator.

- Upon request the last 15 minutes worth of monitor values, along with the limit values may be displayed.

The context diagram, inferred from the requirements, is illustrated in FIGURE 4. The externals are Sensors and Operator. The interfaces between the externals and the software system are identified as flows between them. The flows are labeled with types inferred from the requirements.

\subsection{Example Object DFDs}

The ADOs, represented as bubbles on FIGURE 5, are Monitor_Limit_Sets, which stores the Limit values for each Monitor, and 7 "onitor_History, which stores the last 15 minutes worth of data. The Monitor_Limit_Sets ADO inputs/outputs data of type Limit. The Monitor_History ADO inputs/outputs data of type Monitor. FIGIJE 6 is an example of a declaration diagram ${ }^{1}$ that illustrates the 
Sensor Monitoring System Example

Monitor_History ADO functionality. The definition ADTs, Monitor and Limit, appear on the context diagram. Transforms are needed to construct the definition ADTs, Reports and Displays. The composite flow Displays decomposes into 2 definition ADTs:

$$
\text { Displays }=\text { History_Display }+ \text { Limit_Display }
$$

The transform Detect_Band_Changes is a stand-alone transform, which transforms the Limit values and the current and previous Monitors to a change-of-band Report. The definition ADT Limit_Display includes a construct function that transforms data from type Limit to type Limit_Display. The definition ADT History_Display includes construct functions(s) that transform data from types Limit and Monitor to type History...Display. These ADT transforms are illustrated as anchor points.

\subsection{Example Flow Paths}

There flow paths on the Sensor Monitor System Object DFD are:

1. Display History - This flow path begins with two flows; Monitor out of the ADO Monitor History and Limit out of the ADO Monitor Limit Sets and term; ates with the flow History_Display.

2. Display Limit - This flow path begins with the flow Limit out of the ADO Monitor Limit Sets, and terminates with the flow Limit Display.

3. Update History - This flow path is the single flow labeled Monitor that terminates at the ADO Monitor History.

4. Limit Check - This flow path begins with three flows; The input flow labeled Monitor (Current), the flow labeled Monitor (Previous) out of the ADO Monitor History and the flow labeled Limit out of the ADO Monitor Limit Sets. The flow path includes the transform Detect Band Changes and terminates with the flow Reports.

1. Declaration diagrams are described in Maxted/Rowe [8].

Object-Oriented DFD Models to Present the Functional and Behavioral Views 
5. Update Limit - This flow path is the single flow labeled Limit that terminates at the ADO Monitor Limit Sets.

\subsection{Example Control DFDs}

The control DFDs illustrate the flow of data between actors. In this example, there is one actor for each flow path. Each actor, identified above, is represented as a bubble. The actor invocation parameters are illustrated as flows in and out of the bubble. ADOs are represented as "files". Flows to from the $\mathrm{ADO}$ are used to indicate if an actor accesses the $\mathrm{ADO}$. The control DFD, for the Sensor Monitor System is illustrated in FIGURE 7.

Actors are serialized into threads. If a single thread, Receive_Monitors, is identified to sequentialize the actors Update_History and Limit_Check and a single thread, User_Dialog is identified to coordinate the actors Display_History, Display_Limits, and Update_Limit then the control DFD would be modified. The context diagram would decompose into a DFD illustrating the flow of data through these threads. The threads would then decompose into DFDs illustrating the flow of data between the actors that they sequentialize. This modified hierarchy of control DFDs is not shown. 
Summary

\subsection{Summary}

Categories of software components have been presented. The presented categories expand upon those identified in the current literature. The categories of software components have been introduced because they identify the role of the software component in the DFDs. It has been suggested that further study of the software categories may result in metrics that evaluate the quality of software systems. This is a subject of future work.

An object-oriented design methodology has been presented. DFDs have been employed to present the functional and the behavioral views. These views decompose into actual software cimponents. Combining the views presented herein with a structural view based on Information Models, all the software components are identified. Separating the functional view from the behavioral view simplifies both views. The two views are individually simpler because they repeat instead of intertwine the same story. The functional view and structural view are developed concurrently. The functional view provides the overview identifying the major classes composing a system. The structural view provides the detail structure of these classes. The behavioral view is derived from the functional view. The overlap of components presented in multiple views unites the three views into a more complete specification of the software system. The top-down hierarchical nature of the DFDs provide a much needed overview or road map to the sottware system. This lack of an overview is a frequent complaint of Information Models. The hierarchal overview is valuable to both the customer and the designer, providing a window into the system. Finally, a design document based on these DFDs has been presented.

The methodology presented herein w'as created out of necessity in order to support an object-oriented design of a satellite ground system. An existing DFD CASE tool was employed to design the two views. The functional view presented herein has been used repeatedly, in the past year, during the analysis phase. It provides an overview, valuable during the analysis phase, which compliments the concurrent information modeling. 


\subsection{Acknowledgments}

I wish to express my gratitude to Paul Attermeier, Larry Ellis, Louann Grady, Bruce Malm, and John Rowe from Sandia National Laboratories as well as Sue Spaven of Applied Physic Inc., whose assistance and support were vital to refining and clarifying this methodology.

\subsection{References}

[1] James Rumbaugh, et al, Object-Oriented Modeling and Design, 1991, Prentice Hall

[2] Sally Shlaer, Steven Mellor, Object-Oriented Systems Analysis, Modeling the World in Data, 1988 Prentice-Hall.

[3] Paul T. Ward, Lloyd G. Williams, Domain Analysis: An Example, Software Engineering Research, SERM-013-90, May 1990.

[4] Paul T. Ward, Object-Oriented Requirements Definition, Class presented by NTU Satellite Network on Feb. 11, 1991.

[5] Sally Shlaer, Steven Mellor, Object-Oriented Systems Analysis, Modeling the World in States, 1992 Prentice-Hall.

[6] Mike Frankel, Ada 9X and CASE: A Methods Perspective, CASE World Conference Proceedings, Sept. 30- Oct. 2, 1992, Vol. III, p E29.

[7] Grady Booch, Object-Oriented Design with Applications, 1991 Benjamin/Cummings Publishing

[8] Amelia Maxted, John Rowe, An Ada Graphical Tool to Support Software Development, Ada Letters, Using Ada: ACM SIGAda International Conference, Boston Massachusetts, Dec. 8-11, 1987. 

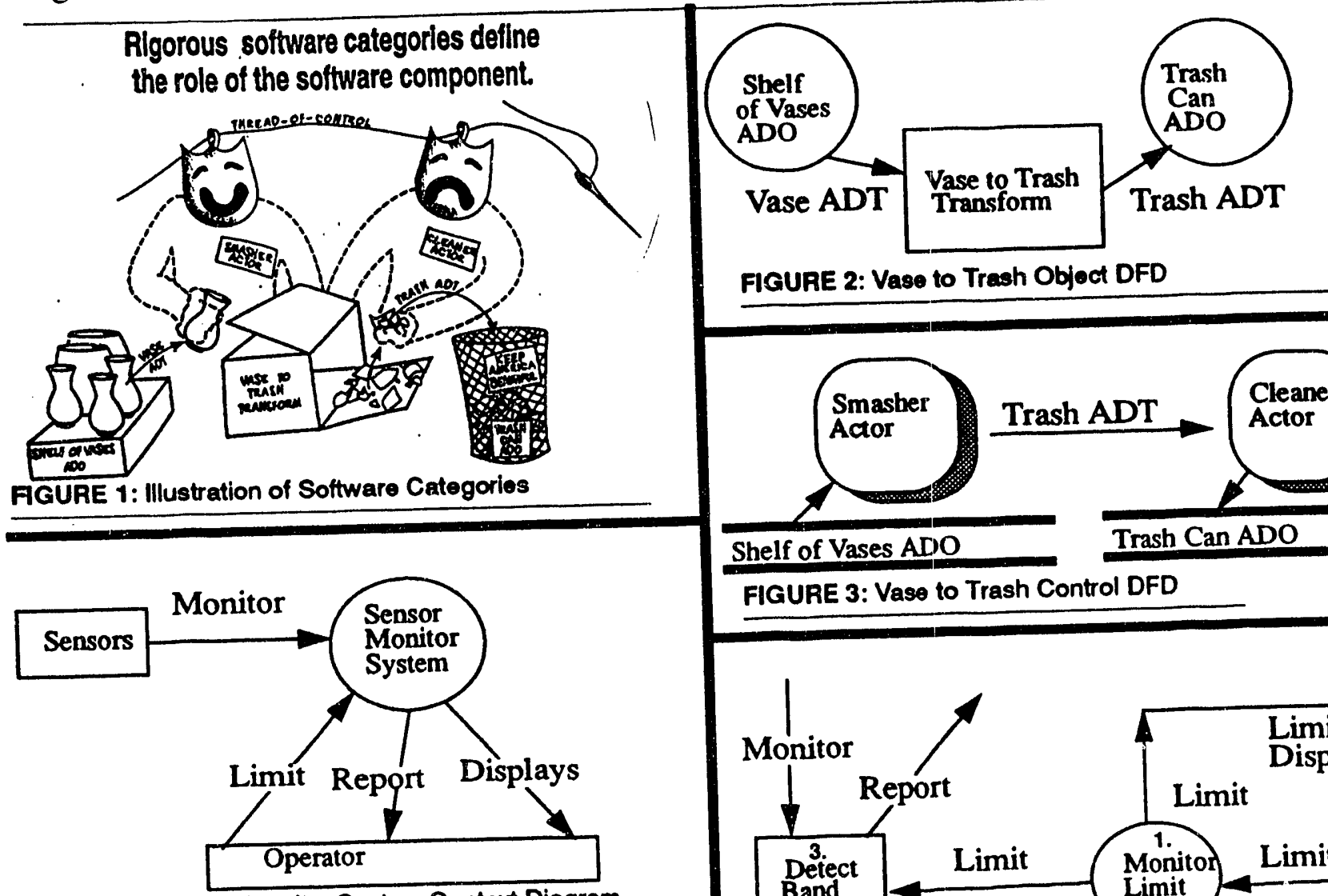

FGURE 4: Sensor Monitor System Context Diagram

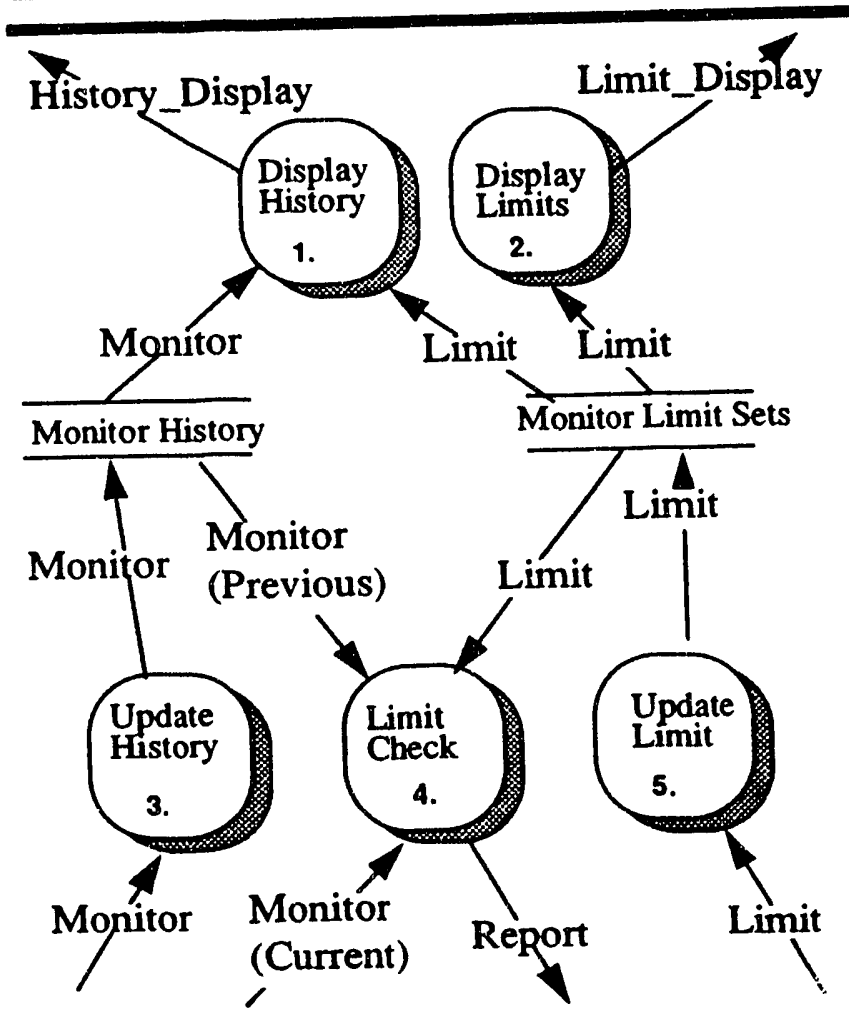

FIGIJRE 7: Sensor Monitor System Control DFD

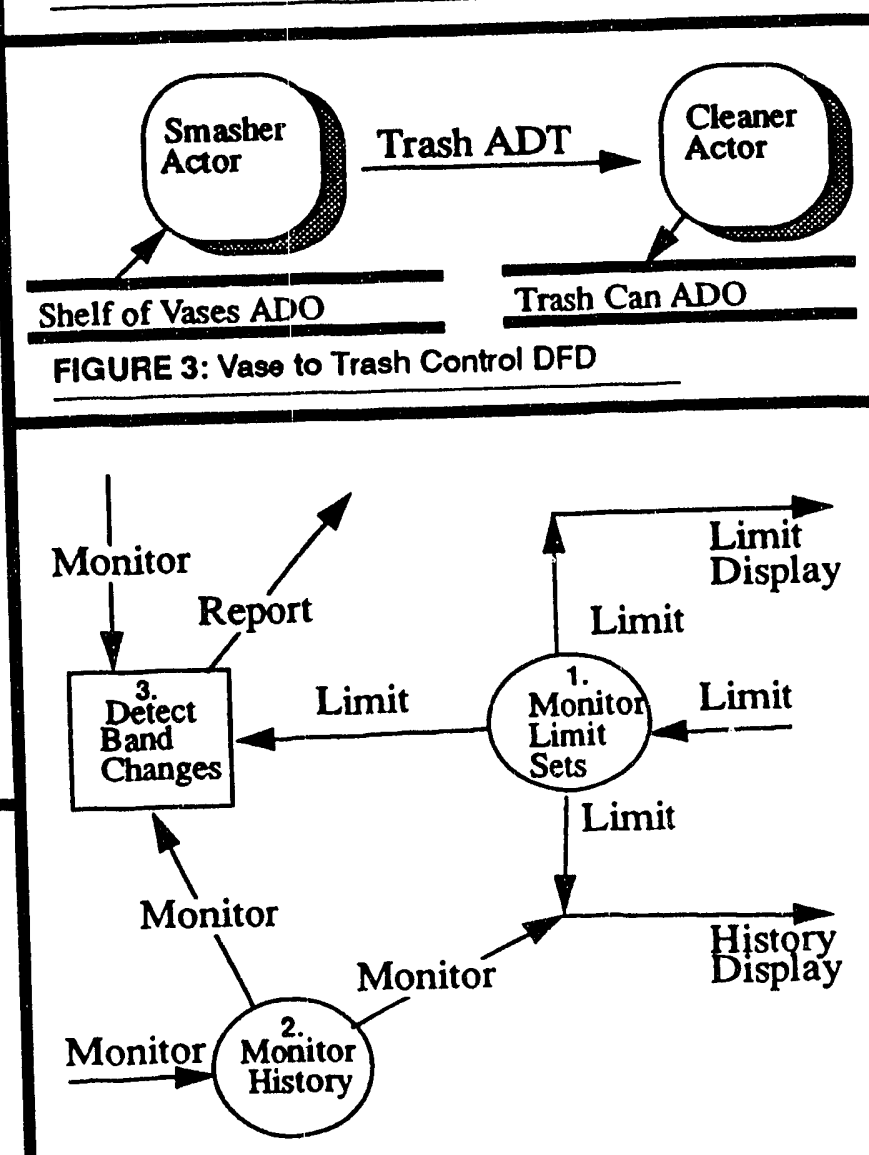

FIGURE 5: Sensor Monitor System Object DFD

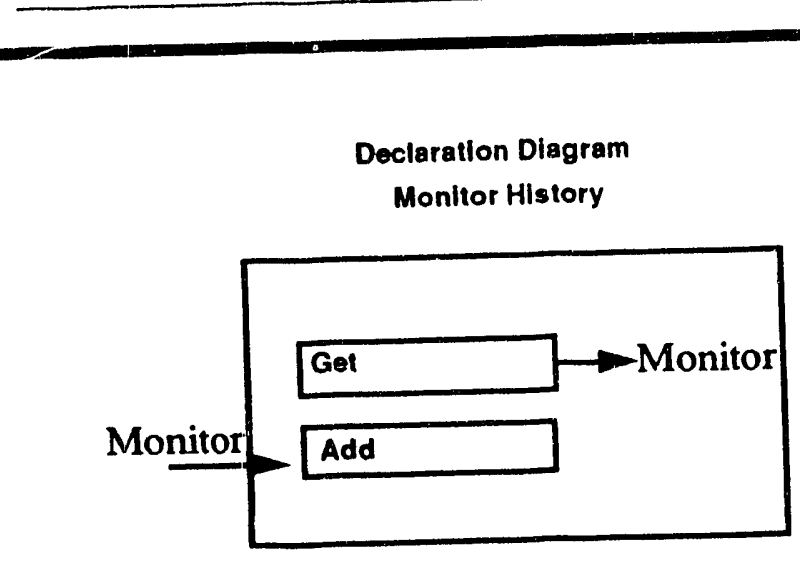

FIGURE 6: Monitor History ADO Declaration Diagram

Object-Oriented DFD Models to Present the Functional and Behavioral Views 


\section{Unlimited Relense}

1 8523-2 Central Technical Files

57141 Technical Library

17151 Technical Publications

10 7613-2 Document Processing for DOE/OSTI

509232 " Amelia Maxted 

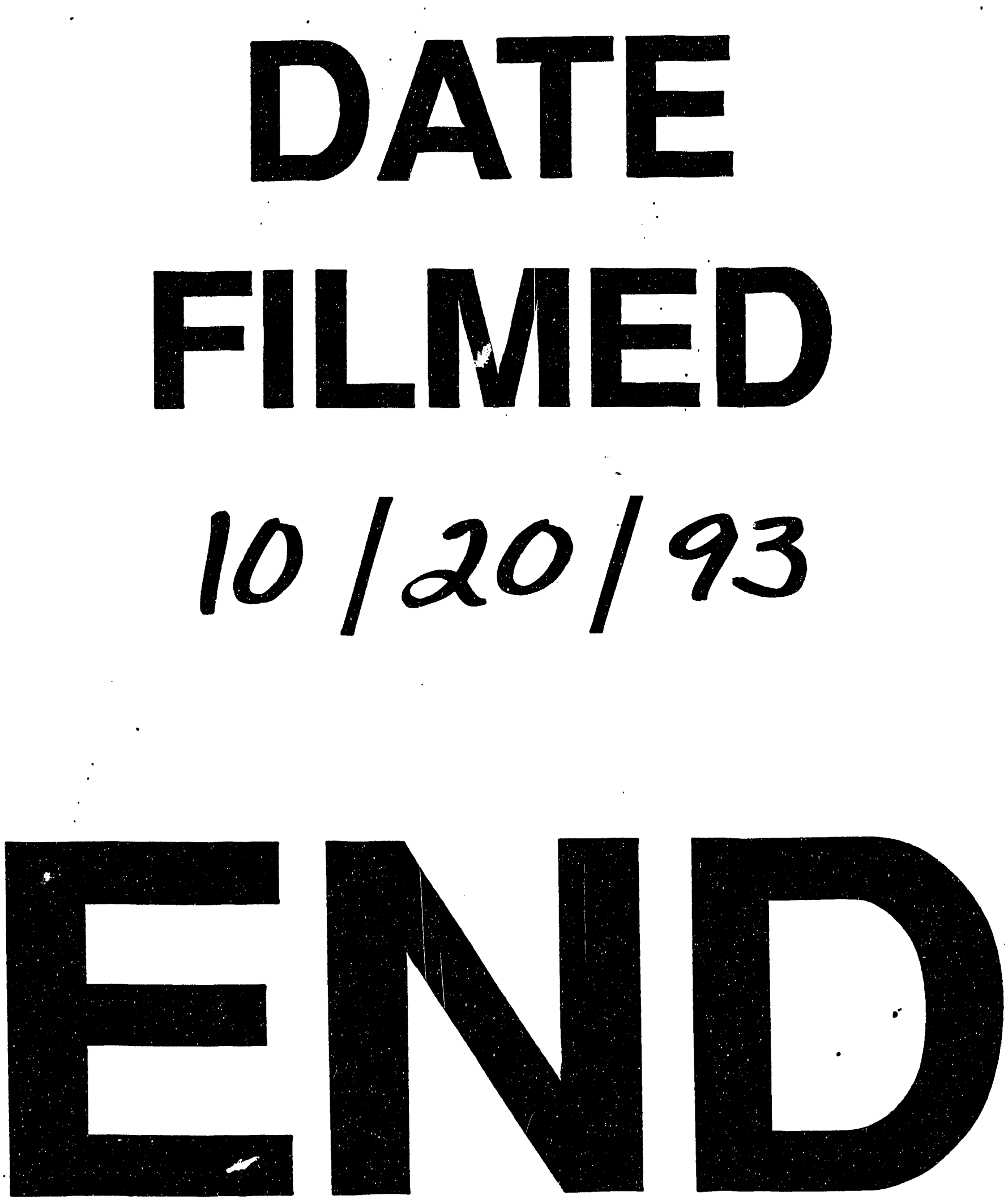
